the idea that preservation of sexual function is more important than improved survival benefit to many patients.

Original article Pietrzak P et al. (2004) Organ-sparing surgery for invasive penile cancer: early follow-up data. $B J U$ Int 94: 1253-1257

\section{Smoking cessation improves erectile dysfunction}

Smoking cessation can decrease the individual's risk of vascular diseases such as coronary artery disease and ischemic stroke. Pourmand et al. hypothesized that the severity of erectile dysfunction would also be reduced. In their recent study, participants who successfully stopped smoking were significantly more likely to show improvements in ED than those who continued.

The study included 118 ex-smokers and 163 current smokers, all of whom had undergone nicotine replacement therapy. All participants reported at least mild erectile dysfunction at the beginning of the study and, besides smoking, had no known risk factors for the condition. The severity of erectile dysfunction was strongly associated with smoking intensity. Although the two groups of participants had similar ED status at the start of the study, those who stopped smoking had a significantly better grade of ED after one year's follow-up. After this period, 30 (25.4\%) of the ex-smokers reported an improvement in their ED status of at least one grade according to the five-item version of the International Index of Erectile Function questionnaire. Those with mild ED at the start of the study showed the greatest improvement, whereas none of those with severe ED improved. Three (2.5\%) individuals in the ex-smokers group experienced a worsening of their condition. In contrast, no individuals in the 'current smokers' group showed an improvement in ED, and $11(6.8 \%)$ reported that their ED status had deteriorated.

In conclusion, the study showed a strong dose-dependent relationship between smoking and ED and demonstrated that smoking cessation can improve the condition.

Original article Pourmand G et al. (2004) Do cigarette smokers with erectile dysfunction benefit from stopping?: a prospective study. BJU Int 94: 1310-1313

\section{Small-cell carcinoma of the bladder: a retrospective study}

Mangar and colleagues from the UK have recently reported their 10-year experience of treating small-cell carcinoma of the urinary bladder. This rare tumor accounts for only $0.5 \%$ of urinary bladder malignancies and so clinical data are scarce.

During the period 1986-1996, the authors treated 14 patients whose median age at presentation was 74 years. All patients presented with stage III or stage IV disease and underwent transurethral resection three were too frail to receive further treatment. Of the remainder, four patients received radical bladder radiotherapy (combined with neoadjuvant chemotherapy in one case), two underwent radical cystoprostatectomy, and five received palliative bladder radiotherapy. Ten (70\%) patients died within 2 years of diagnosis. The median survival was 5 months, or 21 months in those who had received radical treatment. Long-term survival was recorded in only one case; this patient had undergone radical cystoprostatectomy and was alive 7 years later.

Mangar et al. conclude that patients presenting with this tumor are usually elderly, and that treatment is often complicated by frailty and comorbidity. Since local therapy alone did not appear to give good results in this series, the authors recommend platinumbased chemotherapy (followed by bladder radiotherapy if appropriate) in patients who are able to withstand treatment. The majority of patients, however, are too frail for this treatment and the authors acknowledge the role of palliative pelvic radiotherapy in achieving durable symptom control.

\footnotetext{
Original article Mangar SA et al. (2004) Small-cell carcinoma of the urinary bladder: 10-year experience. Clin Oncol 16: 523-527
}

\section{Minimally-invasive treatment for intrinsic sphincter deficiency}

A new study by Tamanini et al. presents 2-year follow-up data on a new, minimally-invasive device for treating stress urinary incontinence secondary to intrinsic sphincter deficiency. Based on the injection of silicon elastomers 